\title{
Non-infectious pediatric uveitis in a single french tertiary center
}

\author{
Dalila Nouar ${ }^{1 *}$, Cyrille Hoarau', Florence Uettwiller ${ }^{2}$, Marie-Laure Le Lez ${ }^{3}$ \\ From 21st European Pediatric Rheumatology (PReS) Congress \\ Belgrade, Serbia. 17-21 September 2014
}

\section{Introduction}

Pediatric uveitis is a blinding disease in $15-20 \%$ of cases. Juvenile idiopathic arthritis (JIA) is the most common disease associated with uveitis (JIAU).Biologics have changed the treatment of uveitis in particular JIAU.

\section{Objectives}

To describe epidemiological, clinical, ophthalmologic and therapeutic characteristic of non infectious pediatric uveitis followed in our center.

\section{Methods}

We retrospectively collected data between 2004 and 2013 for children under 16 years of age followed for non-infectious uveitis in the Department of Ophthalmology and in Pediatric Rheumatology Unit of the Hospital of Tours.

\section{Results}

Of 32 children included there were $62 \%$ of females and the mean age at diagnosis was $7.7 \pm 3.4$ years and $6.5 \pm$ 3.8 years for JIAU. Ophthalmologic complications were present at the diagnosis in $72 \%$ of patients. Uveitis was unilateral in $56 \%$ of cases and anterior in $75 \%$ of cases. At the time of the last follow-up $41 \%$ were idiopathic uveitis and $41 \%$ JIAU. The number of inflammatory cells in the uvea (Tyndall) was improved in $50 \%$ of cases and stabilized in $50 \%$ of cases. One third of patients had presented new ophthalmologic complications. Uveitis was recurrent in $84 \%$ of cases and $75 \%$ of uveitis had relapsed at least once. Local corticosteroid was always used in combination with systemic treatment . Corticotherapy was used in $41 \%$ of cases, and associated to DMARDS (methotrexate) in 19\% of patients. Anti-TNFa (adalimumab) was used in $42 \%$ of patients and $54 \%$ of
JIAU. An improvement in the visual acuity of our cohort was found (4/10 initially versus $6.3 / 10)$. In the subgroup of JIAU (13 patients) visual acuity was modified from 4/ 10 to $5 / 10$ and $23 \%$ patients used the systemic association;corticosteroid and methotrexate , 3 patients relapsed under adalimumab.

\section{Conclusion}

The risk of visual impairment in child uveitis is important. JIA is the main etiology.Biologics prescription have not been validated yet. The lack of consensus on the therapeutic strategy validate the need for a multidisciplinary collaboration between the ophthalmologist and the pediatric rheumatologist for a better management of children.

\section{Disclosure of interest}

None declared.

\section{Authors' details}

${ }^{1}$ Immunology-allergology Unit, Bretonneau University Hospital, Tours, France. ${ }^{2}$ Pediatric Rheumatology Unit, Clocheville University Hospital, Tours, France. ${ }^{3}$ Ophtalmology Unit, Bretonneau University Hospital, Tours, France.

Published: 17 September 2014

doi:10.1186/1546-0096-12-S1-P233

Cite this article as: Nouar et al:: Non-infectious pediatric uveitis in a single french tertiary center. Pediatric Rheumatology 2014 12(Suppl 1): P233. 